\title{
Modeling of Rotational Nonequilibrium in Post-Normal Shock Flow Analyses
}

\author{
Jae Gang Kim* and Iain D. Boyd ${ }^{\dagger}$ \\ University of Michigan, Ann Arbor, MI 48109, USA
}

\begin{abstract}
Recent modeling of thermal nonequilibrium processes in simple molecules like hydrogen and nitrogen has indicated that rotational nonequilibrium becomes as important as vibrational nonequilibrium at high temperature. In this study, to analyze rotational nonequilibrium, the rotational mode is separated from the translational-rotational mode that is usually considered in two-temperature models. Then, the translational, rotational, and electron-electronic-vibrational modes are considered separately in describing the thermochemical nonequilibrium behind a strong normal shock wave. The energy transfer for each energy mode is described by recently evaluated relaxation time parameters including rotation-to-vibration energy transitions and state-to-state kinetics. One-dimensional post-normal shock flow equations are constructed with these thermochemical models, and post-normal shock flow calculations are performed for the conditions of existing shocktube experiments and various re-entry points. In analyzing the shock-tube experiments, it is shown that the present thermochemcial model is able to describe the rotational and electron-electronic-vibrational relaxation processes behind a strong shock wave.
\end{abstract}

\section{Nomenclature}

$\tau \quad$ Relaxation time, sec

$\widetilde{e} \quad$ Average energy per particle, erg

$D \quad$ Dissociation energy of $\mathrm{N}_{2}, \mathrm{erg}$

E Energy, $\mathrm{erg} / \mathrm{g}$

$e \quad$ Energy per particle, erg

$h \quad$ Total enthalpy, $\mathrm{erg} / \mathrm{g}$

$h^{f} \quad$ Species formation energy, $\mathrm{erg} / \mathrm{mol}$

$k \quad$ Boltzmann constant, $\mathrm{erg} / K$

$K(i, j), K(i, c)$ State-to-state transition rates for bound-bound and -free transitions, $\mathrm{cm}^{3} \mathrm{sec}^{-1}$

$K_{f}, K_{r}$ Forward and backward reaction rate coefficient, respectively, $\mathrm{cm}^{3} \mathrm{sec}^{-1}$ or $\mathrm{cm}^{6} \mathrm{sec}^{-1}$

$K_{p}(i, c)$ Predissociation rates, $\sec ^{-1}$

$m \quad$ Species mass, $g$

$n \quad$ Number density, $\mathrm{cm}^{-3}$

$N_{a} \quad$ Avogadro number, $\mathrm{mol}^{-1}$

$p \quad$ Pressure, dyne $/ \mathrm{cm}^{2}$

$Q, Q_{t} \quad$ Internal and translational partition functions

$T, T_{r}, T_{e v}$ Translational, rotational, and electron-electronic-vibrational temperatures, respectively, $K$

u Velocity, $\mathrm{cm} / \mathrm{sec}$

$x \quad$ Distance from shock front, $\mathrm{cm}$

Subscripts

e Electron species

$h \quad$ Heavy particle species

$m \quad$ Molecular species

*Post-doctoral Research Fellow, Department of Aerospace Engineering, 1320 Beal Avenue, Member of AIAA.

$\dagger$ James E. Knott Professor, Department of Aerospace Engineering, 1320 Beal Avenue, AIAA Fellow. 


\section{$r, e v \quad$ Rotational and electron-electronic-vibrational modes, respectively}

\section{Symbols}

$\gamma \quad$ Rovibrational or species concentration, $\mathrm{mol} / \mathrm{g}$

$\rho \quad$ Density, $\mathrm{g} / \mathrm{cm}^{3}$

\section{Introduction}

$\mathrm{T}_{\mathrm{t}}^{\mathrm{H}}$ HE energy modes contained in atoms or molecules are usually characterized by a temperature. In a thermochemical nonequilibrium gas mixture, the temperatures, which characterize these different modes of energy, may be different from each other. Two-temperature and multi-temperature models ${ }^{1}$ are widely used approaches for characterizing these different energy modes. In the two-temperature model, one approximates this situation with two main assumptions. First, one assumes that there are only two different temperatures. The rotational temperature of molecules is assumed to be the same as the translational temperature of heavy particles. Vibrational temperatures of all molecules are assumed to be the same as the electron temperature and the electronic temperature. Second, the forward and reverse rate coefficients for the chemical reactions involving molecules are assumed to be a function of a geometrically averaged temperature evaluated using the translational and vibrational temperatures. In the multi-temperature approach, unlike the two-temperature model, the vibrational temperature is considered as a species dependent characteristic temperature. The electron and electronic temperatures are treated separately from the vibrational temperature. Computational fluid dynamic results obtained from these two- and multi-temperature models show fairly good agreement with experiments at reentry speeds below $8 \mathrm{~km} / \mathrm{sec}$. Recent flight experiments ${ }^{2}$ agree well with the predictions calculated by these temperature models for this reentry speed range.

However, the two-temperature and multi-temperature models are not able to correctly predict the shock stand-off distance for a sphere between Mach numbers of 10 to 15 in air. In the work of Furudate et al., ${ }^{3}$ a comparison of the measured and calculated shock stand-off distances was performed. The measurement was made in a ballistic range in Tohoku University. The calculations were performed using the extended twotemperature model in which the vibrational temperatures of $\mathrm{O}_{2}, \mathrm{~N}_{2}$, and $\mathrm{NO}$ were considered different from each other. In this work, it was shown that the two-temperature model tends to underestimate the shock stand-off distance. This disagreement between the calculated and measured data shows the uncertainties of two- and multi-temperature models and shows that the thermochemical nonequilibrium behind a strong shock wave is not understood well.

One of the uncertainties in modeling high-enthalpy flow is the rotational nonequilibrium behind a strong shock wave. The rotational nonequilibrium behind a strong shock wave were analyzed theoretically in $\mathrm{H}_{2}$ accounting for interactions with $\mathrm{H}, \mathrm{He}$, and $\mathrm{H}_{2}$ by Kim et al. ${ }^{4,5}$ and Kim and Boyd. ${ }^{6}$ The complete sets of the state-to-state transition rates were calculated by a quasi-classical trajectory method using recently calculated ab-initio potential energy surfaces. Analyses were performed using the master equation of postnormal shock and expanding flows with the state-to-state transition rates. It was observed that rotational relaxation at low temperature is faster than for the vibrational modes. However, at high temperatures above $10,000 \mathrm{~K}$, the rotational and vibrational relaxation times are almost identical. In the analysis of post-normal shock and nozzle expanding flows, the rotational mode of $\mathrm{H}_{2}$ exists in a state of nonequilibrium and the calculated rotational temperatures are close to the vibration temperatures.

In $\mathrm{N}_{2}$, the spectrum of the radiation emitted in the flow behind a shock wave was measured and analyzed by several researchers. ${ }^{7,8}$ At the point of peak radiation intensity of the $\mathrm{N}_{2}(2+)$ band, the spectrum was analyzed in detail. From such analyses, it was found that the rotational temperature of $\mathrm{N}_{2}$ is not higher than the vibrational temperature at the peak-intensity point. In a recent measurement using the Coherent Anti-Stokes Raman Spectroscopy (CARS) method, ${ }^{9}$ the temperature of the electronic ground state of $\mathrm{N}_{2}$ was estimated from radiation emitted from the strong shock wave in a free-piston, double-diaphragm shock tube. In this experiment, the estimated rotational temperature is also not higher than the vibrational temperature. The effective collision number of the rotational mode of $\mathrm{N}_{2}$ is the order of 10 . This effective collision number of the rotational mode is much smaller than the vibrational mode. This suggests that the rotational mode can be treated as being in equilibrium with the translational state. However, in the previous shock-tube experiments, the measured rotational temperatures are close to the vibrational temperatures. These rotational nonequilibrium processes of $\mathrm{N}_{2}$ were analyzed theoretically by Kim and Boyd ${ }^{10}$ by full master equation calculations. The complete sets of state-to-state transition rates of $\mathrm{N}_{2}+\mathrm{N}$ were adopted 
from the NASA database. ${ }^{11,12}$ In this work, rotational nonequilibrium is clearly observed. Especially at temperatures above $30,000 \mathrm{~K}$, the relaxation rate of the rotational mode is almost identical to that of the vibrational mode.

In the present work, a thermochemical nonequilibrium model including rotational nonequilibrium is proposed. In treating the rotational nonequilibrium, the rotational mode is separated from the translationalrotational mode of the previous two-temperature model. The other energies, such as electron translation, electronic excitation, and vibrational energies are treated as one, combined mode, the electron-electronicvibrational energy mode. Then, the translational, rotational, and electron-electronic-vibrational modes are employed to describe the thermochemical nonequilibrium behind a strong normal shock wave. The energy transfer for each energy mode is described by recently evaluated relaxation time parameters including rotation-to-vibration energy transitions and state-to-state kinetics. One-dimensional post-normal shock flow equations are constructed with these thermochemical models, and post-normal shock flow calculations are performed for the existing shock-tube experiments and various re-entry conditions.

\section{Thermochemical model including rotational nonequilibrium}

\section{A. 1-D flow equation}

In this study, 22 electronic levels for the $\mathrm{N}$ atom, 15 levels for $\mathrm{N}^{+}, 6$ levels for $\mathrm{N}_{2}$, and 5 levels for $\mathrm{N}_{2}^{+}$are taken into account. The rovibrational energies for each electronic state are calculated from the Dunham expansion model. The spectral data for this model are obtained from the NIST database. However, the rovibrational energies of ground state $\mathrm{N}_{2}\left(X^{1} \Sigma_{g}^{+}\right)$are treated exactly. ${ }^{11,12}$ This results in 9,390 rovibrational states, that include both truly bound and quasi-bound states. The post-normal shock flow field is described by a one-dimensional flow assumption. Then the mass, momentum, and energy conservation equations in the steady-state flow conditions are derived as

$$
\begin{aligned}
\frac{1}{\rho} \frac{d \rho}{d x} & =-\frac{1}{u^{2}} \frac{d}{d x}\left(\frac{u^{2}}{2}\right), \\
\frac{d}{d x}\left(\frac{u^{2}}{2}\right) & =-\frac{1}{\rho} \frac{d p}{d x}, \\
u \frac{d h}{d x} & =0,
\end{aligned}
$$

where pressure and enthalpy are defined as

$$
\begin{gathered}
p=\sum_{s}^{h} \rho N_{a} \gamma_{s} k T+\rho N_{a} \gamma_{e} k T_{e v}, \\
h=\sum_{s}^{a l l} h_{s}^{f} \gamma_{s}+\sum_{s}^{h} \frac{5}{2} N_{a} k T \gamma_{s}+N_{a} \gamma_{e} k T_{e v}+E_{e v}+E r+\frac{u^{2}}{2},
\end{gathered}
$$

respectively.

\section{B. Chemical reaction model}

The chemical reactions of heavy particle and electron impact dissociation $\left(\omega_{D 1}-\omega_{D 5}\right)$, predissociation $\left(\omega_{D 6}\right)$, electron impact ionization $\left(\omega_{E I}\right)$, associative ionization $\left(\omega_{A I}\right)$, charge exchange reaction $\left(\omega_{C E}\right)$, and radiative recombination $\left(\omega_{R R}\right)$ for 5 species are considered in the present work. Except for the dissociation reaction $\mathrm{N}_{2}+\mathrm{N}$ and the predissociation of $\mathrm{N}_{2}$, all chemical reactions are treated by Arrhenious-type rate coefficients. The Arrhenious reaction rate is defined as

$$
K_{f}\left(T_{f}\right)=C T_{f}^{n} \exp \left(-\theta / k T_{f}\right) .
$$

The backward reaction rate is evaluated from the equilibrium constant:

$$
K_{b}\left(T_{b}\right)=K_{f}\left(T_{b}\right) / K_{e}\left(T_{b}\right) .
$$


Table 1. Reaction rate coefficients

\begin{tabular}{cccccccc}
\hline \hline Reaction & $M$ & $T_{f}$ & $T_{b}$ & $C$ & $n$ & $\theta$ & index \\
\hline $\mathrm{N}_{2}+\mathrm{M} \rightarrow \mathrm{N}+\mathrm{N}+\mathrm{M}$ & $\mathrm{N}_{2}$ & $\sqrt{T T_{e v}}$ & $T$ & $7.0^{21}$ & -1.60 & 113,200 & $\omega_{D 2}$ \\
& $\mathrm{~N}^{+}$ & $\sqrt{T T_{e v}}$ & $T$ & $3.0^{22}$ & -1.60 & 113,200 & $\omega_{D 3}$ \\
& $\mathrm{~N}_{2}^{+}$ & $\sqrt{T T_{e v}}$ & $T$ & $7.0^{21}$ & -1.60 & 113,200 & $\omega_{D 4}$ \\
& $\mathrm{e}^{-}$ & $T_{e v}$ & $T_{e v}$ & $1.2^{25}$ & -1.60 & 113,200 & $\omega_{D 5}$ \\
$\mathrm{~N}+\mathrm{N} \rightarrow \mathrm{N}_{2}^{+}+\mathrm{e}^{-}$ & & $T$ & $T$ & $4.4^{7}$ & 1.5 & 67,500 & $\omega_{A I}$ \\
$\mathrm{~N}^{+}+\mathrm{N}_{2} \rightarrow \mathrm{N}_{2}^{+}+\mathrm{N}$ & & $T$ & $T$ & $1.0^{12}$ & 0.5 & 12,200 & $\omega_{C E}$ \\
$\mathrm{~N}+\mathrm{e}^{-} \rightarrow \mathrm{N}^{+}+\mathrm{e}^{-}+\mathrm{e}^{-}$ & & $T_{e v}$ & $T_{e v}$ & $2.5^{34}$ & -3.82 & 168,600 & $\omega_{E I}$ \\
$\mathrm{~N}^{+}+\mathrm{e}^{-} \rightarrow \mathrm{N}+h \nu$ & & $T_{e v}$ & $T_{e v}$ & $1.52^{11}$ & -0.48 & & $\omega_{R R}$ \\
\hline \hline
\end{tabular}

The reaction rate parameters $C, n$, and $\theta$ are obtained from Park's work, ${ }^{13}$ and these parameters are tabulated in Table 1. In the present work, rotational nonequilibrium is not considered in these Arrhenious-type chemical reactions. This is because there does not exist a model to describe the rotational nonequilibrium effect in these chemical reactions. However, in the dissociation of $\mathrm{N}_{2}+\mathrm{N}$ and the predissociation of $\mathrm{N}_{2}$, the rotational and vibrational nonequilibrium effects are fully considered by coupling the system of master equations. These dissociation and predissociation processes are evaluated by using the following equations;

$$
\begin{gathered}
\omega_{D 1}=\sum_{i} K(i, c)\left[\gamma_{i}-\frac{Q_{i} Q_{t_{N_{2}}}}{Q_{N}^{2} Q_{t_{N}}^{2}} \exp \left(\frac{D_{i}}{k T}\right) \rho N_{a} \gamma_{N}^{2}\right] \rho N_{a} \gamma_{N} \\
\omega_{D 6}=\sum_{i} K_{p}(i, c)\left[\gamma_{i}-\frac{Q_{i} Q_{t_{N_{2}}}}{Q_{N}^{2} Q_{t_{N}}^{2}} \exp \left(\frac{D_{i}}{k T}\right) \rho N_{a} \gamma_{N}^{2}\right],
\end{gathered}
$$

where index $i$ is the rovibrational state of ground state $\mathrm{N}_{2}$. The state-to-state transition rates for bound-free transitions $K(i, c)$ and predissociation $K_{p}(i, c)$ are obtained from the NASA database. ${ }^{11,12}$ Then, the species conservation equations for the 4 heavy species are constructed as

$$
\begin{gathered}
u \frac{d \gamma_{N}}{d x}=2\left(\omega_{D 1}+\omega_{D 2}+\omega_{D 3}+\omega_{D 4}+\omega_{D 5}+\omega_{D 6}\right)-2 \omega_{A I}-\omega_{E I}+\omega_{C E}+\omega_{R R}, \\
u \frac{d \gamma_{N_{2}}}{d x}=-\left(\omega_{D 1}+\omega_{D 2}+\omega_{D 3}+\omega_{D 4}+\omega_{D 5}+\omega_{D 6}\right)-\omega_{C E} \\
u \frac{d \gamma_{N^{+}}}{d x}=\omega_{E I}-\omega_{C E}-\omega_{R R} \\
u \frac{d \gamma_{N_{2}^{+}}}{d x}=\omega_{A I}+\omega_{C E} \\
u \frac{d \gamma_{e^{-}}}{d x}=\omega_{E I}+\omega_{A I}-\omega_{R R} .
\end{gathered}
$$

\section{Internal Energy Exchange Models}

In describing the thermochemical nonequilibrium of $\mathrm{N}, \mathrm{N}_{2}, \mathrm{~N}^{+}, \mathrm{N}_{2}^{+}$, and $\mathrm{e}-$, three pools of energy are considered consisting of the translational, rotational, and electron-electronic-vibrational energies modes. The energy transfer among these energy modes is calculated by Landau-Teller type equations. However, the rotational and vibrational energy transfer for $\mathrm{N}_{2}+\mathrm{N}$ is calculated by coupling the full master equations. Then, the rotational and electron-electronic-vibrational relaxations including the energy loss and gain due to chemical reactions are evaluated by using the following equations;

$$
u \frac{d E_{r}}{d x}=-\sum_{s}^{m} N_{a} \gamma_{e} \frac{2 m_{e}}{m_{s}} g_{r, s} v_{s} \frac{3}{2} k\left(T_{r}-T_{e v}\right)
$$




$$
\begin{aligned}
& +\sum_{i}^{N_{2}} \sum_{j}^{N_{2}} e_{r_{N_{2}}}(i) K(i, j)\left[\frac{Q_{i}}{Q_{j}} \gamma_{j}-\gamma_{i}\right] \rho N_{a}^{2} \gamma_{N} \\
& +\sum_{s}^{m}\left[\frac{\widetilde{e_{r, s}}(T) N_{a} \gamma_{s}-E_{r, s}}{\tau_{r, s}}+f_{R V, s} \frac{E_{v, s}-\widetilde{e_{v, s}}\left(T_{r}\right) N_{a} \gamma_{s}}{\tau_{v, s}}\right] \\
& -\sum_{i}^{N_{2}} e_{r_{N_{2}}}(i) K(i, c)\left[\gamma_{i}-\frac{Q_{i} Q_{t_{N_{2}}}}{Q_{N}^{2} Q_{t_{N}}^{2}} \exp \left(\frac{D_{i}}{k T}\right) \rho N_{a} \gamma_{N}^{2}\right] \rho N_{a}^{2} \gamma_{N} \\
& -\sum_{i}^{N_{2}} e_{r_{N_{2}}}(i) K_{p}(i, c)\left[\gamma_{i}-\frac{Q_{i} Q_{t_{N_{2}}}}{Q_{N}^{2} Q_{t_{N}}^{2}} \exp \left(\frac{D_{i}}{k T}\right) \rho N_{a} \gamma_{N}^{2}\right] N_{a} \\
& \text { - } \Psi_{r} D_{N_{2}} N_{a}\left(\omega_{D 2}+\omega_{D 3}+\omega_{D 4}+\omega_{D 5}\right) \text {, } \\
& u \frac{d E_{e v}}{d x}=\sum_{s}^{h} N_{a} \gamma_{e} \frac{2 m_{e}}{m_{s}} v_{s} \frac{3}{2} k\left(T-T_{e v}\right)+\sum_{s}^{m} N_{a} \gamma_{e} \frac{2 m_{e}}{m_{s}} g_{r, s} v_{s} \frac{3}{2} k\left(T_{r}-T_{e v}\right) \\
& +\sum_{i}^{N_{2}} \sum_{j}^{N_{2}} e_{v_{N_{2}}}(i) K(i, j)\left[\frac{Q_{i}}{Q_{j}} \gamma_{j}-\gamma_{i}\right] \rho N_{a}^{2} \gamma_{N} \\
& +\sum_{s}^{m}\left[\left(1-f_{R V, s}\right) f_{D} \frac{\widetilde{e_{v, s}}(T) N_{a} \gamma_{s}-E_{v, s}}{\tau_{v, s}}+f_{R V, s} \frac{\widetilde{e_{v, s}}\left(T_{r}\right) N_{a} \gamma_{s}-E_{v, s}}{\tau_{v, s}}\right] \\
& -\sum_{i}^{N_{2}} e_{v_{N_{2}}}(i) K(i, c)\left[\gamma_{i}-\frac{Q_{i} Q_{t_{N_{2}}}}{Q_{N}^{2} Q_{t_{N}}^{2}} \exp \left(\frac{D_{i}}{k T}\right) \rho N_{a} \gamma_{N}^{2}\right] \rho N_{a}^{2} \gamma_{N} \\
& -\sum_{i}^{N_{2}} e_{v_{N_{2}}}(i) K_{p}(i, c)\left[\gamma_{i}-\frac{Q_{i} Q_{t_{N_{2}}}}{Q_{N}^{2} Q_{t_{N}}^{2}} \exp \left(\frac{D_{i}}{k T}\right) \rho N_{a} \gamma_{N}^{2}\right] N_{a} \\
& \text { - } \Psi_{v} D_{N_{2}} N_{a}\left(\omega_{D 2}+\omega_{D 3}+\omega_{D 4}+\omega_{D 5}\right)-D_{N_{2}} N_{a} \omega_{D 5}-I_{N} N_{a} \omega_{E I} .
\end{aligned}
$$

In the present work, the electron-rotational energy transitions are described by the model proposed by Nishida and Matsumoto, ${ }^{14}$ and the coefficient $g_{r, s}$ is set to a constant of 10.0 for neutral and charged species. In electron-translation transitions, the collision frequency $v_{s}$ is calculated by the following equation as

$$
v_{s}=n_{s} \sigma_{e, s}\left(\frac{8 k T_{e v}}{\pi m_{e}}\right)^{1 / 2}
$$

where the effective cross section $\sigma_{e, s}$ for neutral species is obtained from a curve-fit value proposed by Gnoffo et al. ${ }^{15}$ For charged species, the effective cross sections with the Debye cut-off approximation ${ }^{1}$ are adopted in the present work.

$$
\sigma_{e, s}=\frac{4}{3} \frac{4.39 \times 10^{-6}}{T_{e v}^{2}} \ln \left(\frac{1.24 \times 10^{4} T_{e v}^{1.5}}{\sqrt{n_{e}}}\right) .
$$

In describing the rotation-to-vibration transitions, Park's model ${ }^{16}$ is adopted with some modifications. In Park's model, the fractional contribution of the rotation-to-vibration energy transfer to the total energy transfer is set to a constant of 0.4 and this value is derived from $f_{R V}=k T /(k T+1.5 k T)$. In the present work, unlike Park's model, the fractional contribution $f_{R V}$ for species $s$ is determined by

$$
f_{R V, s}=\frac{\left(\xi_{v, s} / 2\right) k T}{\left(\xi_{r, s} / 2\right) k T+1.5 k T},
$$

where $\xi_{r, s}$ and $\xi_{v, s}$ are the numbers of degrees of freedom of the rotational and vibrational modes, respectively. In the energy loss and gain due to chemical reactions, the normalized energy loss ratios, $\Psi_{r}$ and $\Psi_{v}$, are set to constants of 0.35 and 0.5 , respectively. From the recent master equation studies ${ }^{6,10}$ for $\mathrm{H}_{2}$ and $\mathrm{N}_{2}$, the energy losses approach values of 0.35 and 0.5 for the rotational and vibrational modes, respectively. The 


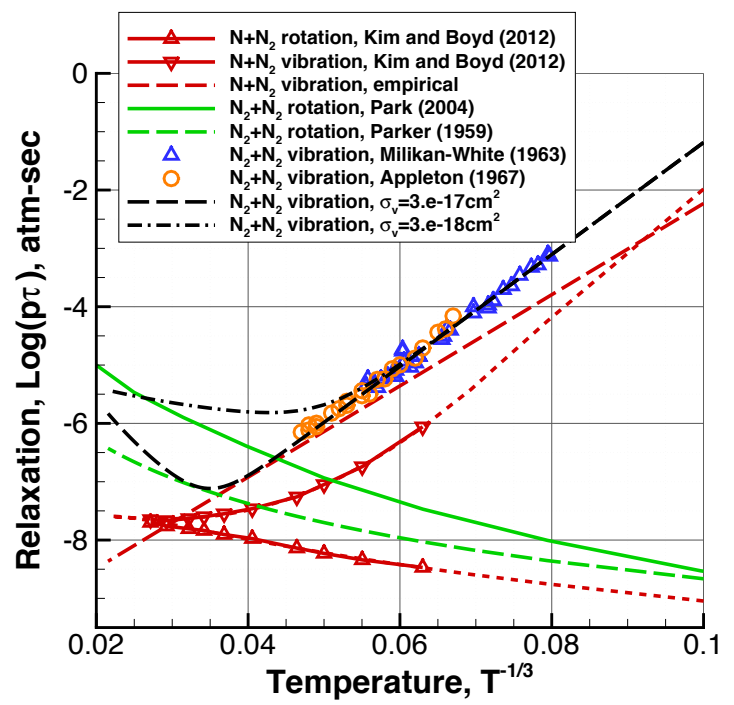

Figure 1. Comparisons of rotational and vibrational relaxations between $\mathbf{N}+\mathbf{N}_{2}$ and $\mathbf{N}_{2}+\mathbf{N}_{2}$.

diffusion correction factor ${ }^{1} f_{D}$ in vibration-to-translation transfer is defined as

$$
f_{D}=\left|\frac{T_{s}-T_{e v}}{T_{s}-T_{v s}}\right|^{s-1}
$$

where $s$ is an arbitrary parameter given as $3.5 \times \exp \left(-5,000 / T_{s}\right)$ for $\mathrm{N}_{2}$ and $\mathrm{N}_{2}^{+} . T_{s}$ and $T_{v s}$ are the translational and electron-electronic vibrational temperatures immediately behind the shock wave. In Eqs. (15) and (16), the unknown variable is the rovibrational concentration $\gamma_{i}$. Unfortunately, we cannot directly calculate $\gamma_{i}$ from the state-to-state kinetics, because of the lack of knowledge about the state-to-state transition rates for $\mathrm{N}_{2}+\mathrm{N}_{2}$. In the present work, this rovibrational concentration $\gamma_{i}$ is determined from the Boltzmann distribution according to the nonequilibrium temperatures as follows;

$$
\gamma_{i}=\frac{Q_{i}\left(T_{r}, T_{e v}\right)}{\sum_{i} Q_{i}\left(T_{r}, T_{e v}\right)} \gamma_{N_{2}}
$$

In Fig. 1, the rotational and vibrational relaxation parameters for $\mathrm{N}+\mathrm{N}_{2}$ and $\mathrm{N}_{2}+\mathrm{N}_{2}$ are compared. In the rotational relaxations for $\mathrm{N}_{2}+\mathrm{N}_{2}$, the relaxation time $\tau_{r}$ was evaluated theoretically by Parker ${ }^{17}$ using the rigid-rotor assumption. This relaxation time is much faster than the relaxation time proposed by Park ${ }^{16}$ that was obtained from the existing state-to-state rotational transition rates using the expression by Rahn and Palmer. ${ }^{18}$ In the present work, the relaxation time by Park is adopted to calculate the rotation-totranslation energy transition for molecule-molecule collisions. The rotational relaxation time parameter by Park is derived as $p \tau_{r}=2.47 \times 10^{-14} T^{1.692} \mathrm{~atm}-\mathrm{sec}$. For molecule-atom collisions, the rotational relaxation time $\tau_{r}$ evaluated by the master equation calculations ${ }^{10}$ for $\mathrm{N}_{2}+\mathrm{N}$ is adopted. In the vibrational relaxation for $\mathrm{N}_{2}+\mathrm{N}_{2}$, the collision limited relaxation time ${ }^{1} \tau_{c}$ is added to the Millikan-White relaxation time ${ }^{19} \tau_{M W}$ to account for the fact that the vibrational excitation rates can not exceed the elastic collision rates. This collision-limited relaxation time is defined as

$$
\begin{gathered}
\tau_{c}=\left(n_{t} \sigma_{v} \sqrt{\frac{8 k T}{\pi m_{r}}}\right)^{-1}, \\
\sigma_{v}=\sigma_{v}^{*}\left(\frac{50,000}{T}\right)^{2}
\end{gathered}
$$

where $n_{t}$ and $m_{r}$ are the total number density of colliding particles and the average mass of the mixture, respectively. In Eq. (23), $\sigma_{v}{ }^{*}$ varies from $\sim 10^{-16}$ to $\sim 10^{-18} \mathrm{~cm}^{2}$. The widely used value is $3.0 \times 10^{-17} \mathrm{~cm}^{2}$. 


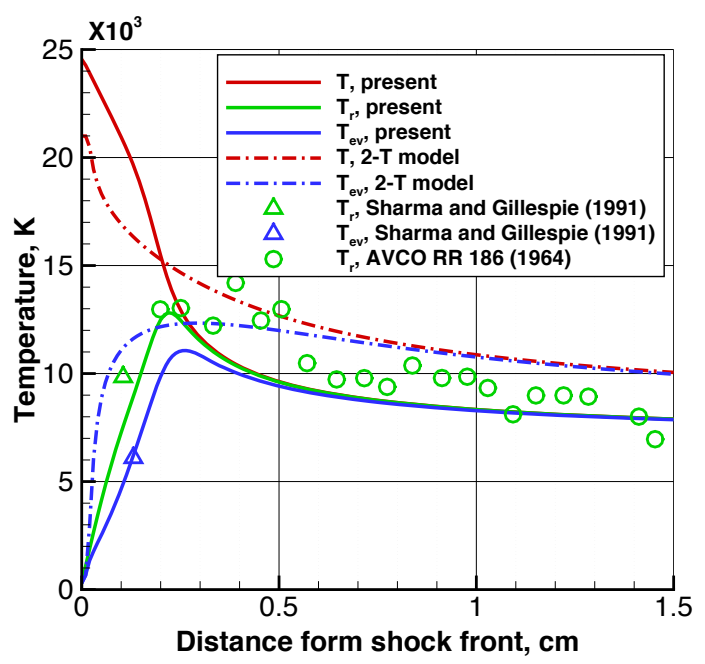

(a) Rotational and electron-electronic-vibrational temperatures.

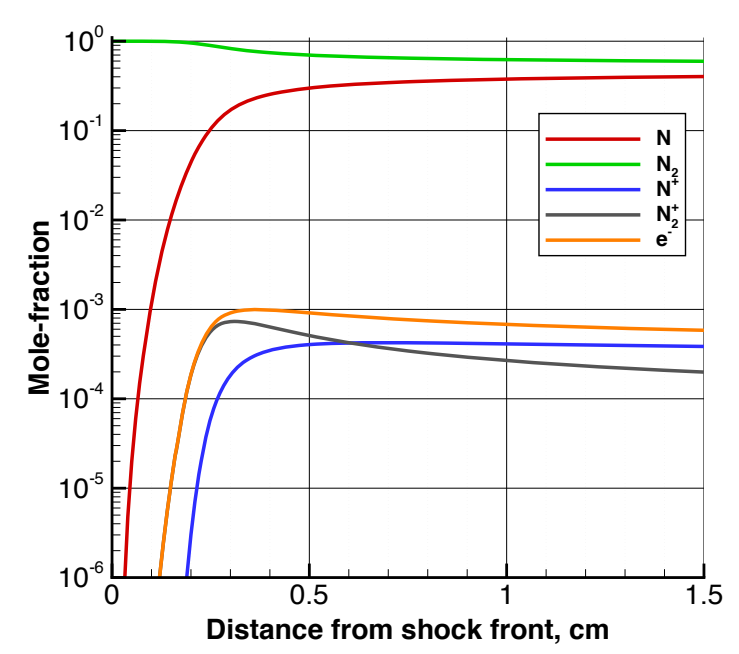

(b) Species mole-fractions.

Figure 2. Comparisons of the present rotational and electron-electronic-vibrational temperatures and species mole-fractions with the shock-tube data by Sharma and Gillespie ${ }^{7}$ and AVCO. ${ }^{21}$

However, the vibrational relaxation time with the collision limiting cross section of $3.0 \times 10^{-17} \mathrm{~cm}^{2}$ is much faster than that of Park's rotational relaxation model ${ }^{16}$ above $10,000 \mathrm{~K}$. In the previous master equation studies for $\mathrm{H}_{2}{ }^{6}$ and $\mathrm{N}+\mathrm{N}_{2},{ }^{10}$ it was observed that the vibrational and rotational relaxation times become identical when the temperature increases. In the work by Kim and Boyd ${ }^{10}$ the collision limiting cross section $\sigma_{v}$ was modified to a constant of $3.0 \times 10^{-18} \mathrm{~cm}^{2}$ to satisfy these relaxation patterns. This cross section was determined through comparisons with the shock-tube data of the Millikan and White ${ }^{19}$ and Appleton. ${ }^{20}$ In the present work, these Millikan-White relaxation parameters with the modified collision limiting cross sections are adopted in describing the vibration-to-translation energy transitions for moleculemolecule collisions. In vibration-to-translation energy transitions for molecule-atom collisions, the vibrational relaxation time evaluated from the master equation calculations ${ }^{10}$ for $\mathrm{N}_{2}+\mathrm{N}$ is adopted.

\section{Post-normal shock flow calculations}

A one-dimensional post-normal shock flow code is developed by using the present thermochemical nonequilibrium model including rotational nonequilibrium. The flow code is constructed in two parts; in the first part, the downstream conditions are determined by using the Rankine-Hugoniot relations for a frozen flow. In the second part, the thermochemical nonequilibrium flows are calculated by an implicit integration method. In the present work, the post-normal shock flow analyses are carried out for existing shock-tube experiments and various re-entry conditions by using this post-normal shock flow code.

In Fig. 2, comparisons of the computed rotational and electron-electronic vibrational temperatures and species mole-fractions with the shock-tube experiments by Sharma and Gillespie ${ }^{7}$ and $\mathrm{AVCO}^{21}$ are presented. In the 1-D post-normal shock calculations, the ambient pressure is set to 1.0 Torr and the shock velocity is $6.2 \mathrm{~km} / \mathrm{sec}$. The free-stream temperature is $300 \mathrm{~K}$. In this case, the total enthalpy of the free-stream is about $20 \mathrm{MJ} / \mathrm{kg}$ and the density is $1.497 \times 10^{-6} \mathrm{~g} / \mathrm{cm}^{3}$. In the present work, energy-equivalent rotational and electron-electronic-vibrational temperatures are adopted to characterize the rotational and electronelectronic-vibrational modes. In figure (a), in the present calculation, the translational temperature behind the normal shock is about 24,300 K and the rotational and electron-electronic-vibrational temperatures agree well with the temperatures measured by Sharma and Gillespie. After converging to the equilibrium temperature, where the distance is $0.3 \mathrm{~cm}$, the present temperature is slightly underestimated compared to the AVCO data. However, the present results show more reasonable results for the rotational and vibrational nonequilibrium in the post-normal shock flows than the results of the two-temperature model. In the twotemperature model, the translational-rotational temperature behind the shock is about $21,000 \mathrm{~K}$ and the 


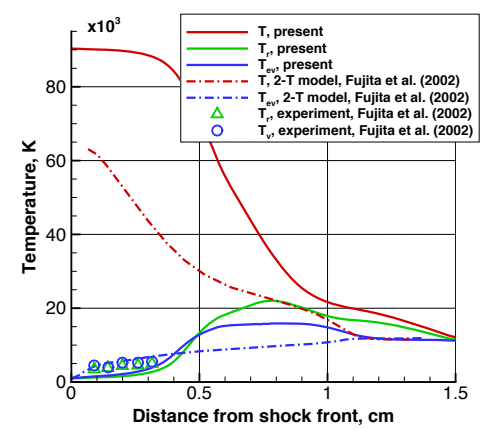

(a) Rotational and electron-electronicvibrational temperatures.

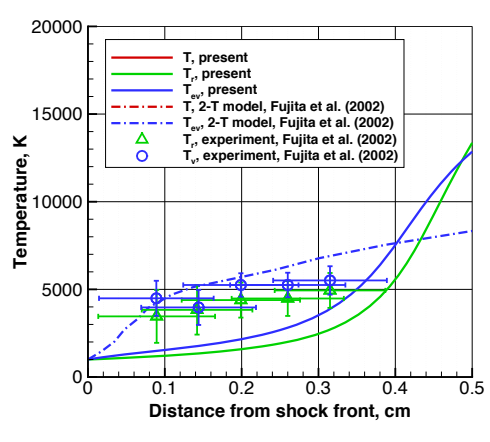

(b) Comparisons of the calculated temperatures with measured data

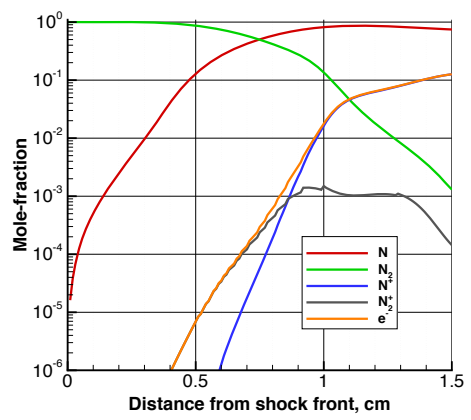

(c) Species mole-fractions.

Figure 3. Comparisons of the present rotational and electron-electronic-vibrational temperatures and species mole-fractions with the shock-tube data by Fujita et al. ${ }^{8}$

vibrational temperature is significantly higher than the measured vibrational temperature. In figure (b), it is shown that the dissociation of $\mathrm{N}_{2}$ occurs rapidly from $0.1 \mathrm{~cm}$ to $0.3 \mathrm{~cm}$ in the present calculation. This is because collision of $\mathrm{N}$ triggers the dissociation of $\mathrm{N}_{2}$ much more than the collision of $\mathrm{N}_{2}$. The mole fractions of electrons and $\mathrm{N}_{2}^{+}$are increased by the associative ionization reactions of $\mathrm{N}+\mathrm{N}$, and then $\mathrm{N}_{2}^{+}$is slightly decreases due to the charged exchange reaction.

In Fig. 3, the computed rotational and electron-electronic vibrational temperatures and species molefractions are compared with the shock-tube experiments by Fujita et al. ${ }^{8}$ In the shock-tube experiments, air radiation from behind strong shock waves was measured for the $\mathrm{N}_{2}(2+)$ system using a free-piston doublediaphragm shock tube. The spatial variation of radiation spectra was obtained using spatially resolved imaging spectroscopy at a shock velocity of $11.9 \mathrm{~km} / \mathrm{sec}$ in the ambient pressure at 0.3 Torr. A series of point wise spectroscopy analysis was carried out in order to obtain a spatial profile of temperatures. In the post-normal shock flow calculations, the freestream conditions are set to the shock-tube experimental conditions. In figure (a), the rotational and electron-electronic vibrational temperatures of the present calculation are almost identical until the distance of $0.5 \mathrm{~cm}$. Beyond the distance of $0.5 \mathrm{~cm}$, the rotational temperature is slightly higher than the vibrational temperatures and it converges more rapidly to the equilibrium temperature than the electron-electronic vibrational mode. This is because the rotational relaxation is slightly faster than the vibrational relaxation in this temperature range. In comparison with the measured temperatures of figure (b), the present nonequilibrium temperatures are lower. However, the rates of increasing temperature are similar to the measured temperatures. The measured rotational temperatures of $\mathrm{N}_{2}$ are similar to the vibrational temperatures, and the present calculated rotational temperatures are also close to the electron-electronic-vibrational temperatures. These results show that strong rotational nonequilibrium exists behind the shock wave, and the present thermochemical model can capture this phenomenon. However, in the two-temperature model, even though the calculated vibrational temperature is similar to the measured temperature, the rotational temperature is totally different from the measured rotational temperature. In the mole-factions of figure(c), it is found that the mole fraction of $\mathrm{N}$ atoms increases rapidly between the distance of $0.5 \mathrm{~cm}$ and $1.0 \mathrm{~cm}$. This means that the rotational and electron-electronic vibrational relaxations occur mainly through $\mathrm{N}_{2}+\mathrm{N}$ collisions. The $\mathrm{m}$ ole fractions of $\mathrm{N}^{+}$and $\mathrm{E}-$ also rapidly increase between $0.5 \mathrm{~cm}$ to $1.0 \mathrm{~cm}$ through the associative ionization and charge exchange reactions.

In Fig. 4, comparisons of the computed rotational and electron-electronic vibrational temperatures and species mole-fractions with the shock-tube experiments by Sakurai et $\mathrm{al}^{9}$ are presented. In the shock-tube experiment, the CARS method was employed to measure the temperatures of the ground state from the radiation behind the strong shock wave. ND:YAG and dye lasers were used in this method. When these lasers excited the nitrogen molecules behind the shock wave, the CARS signal was collected by a spectrograph. Then, the rotational and vibrational temperatures were estimated using a spectral matching method. In this shock-tube experiment by CARS, the rotational and vibrational temperatures were measured at the shock wave velocity of $7.6 \mathrm{~km} / \mathrm{sec}$ and ambient pressure of 0.25 Torr. In the present work, the post-normal shock flow calculations are performed for these conditions. In figure (a), the calculated rotational and electronelectronic-vibrational temperatures rapidly increase behind the shock wave for this relatively high ambient 


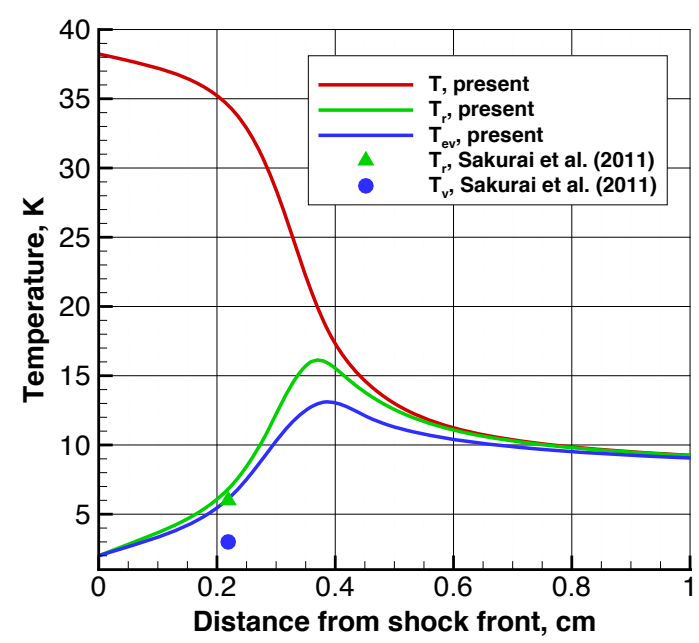

(a) Rotational and electron-electronic-vibrational temperatures.

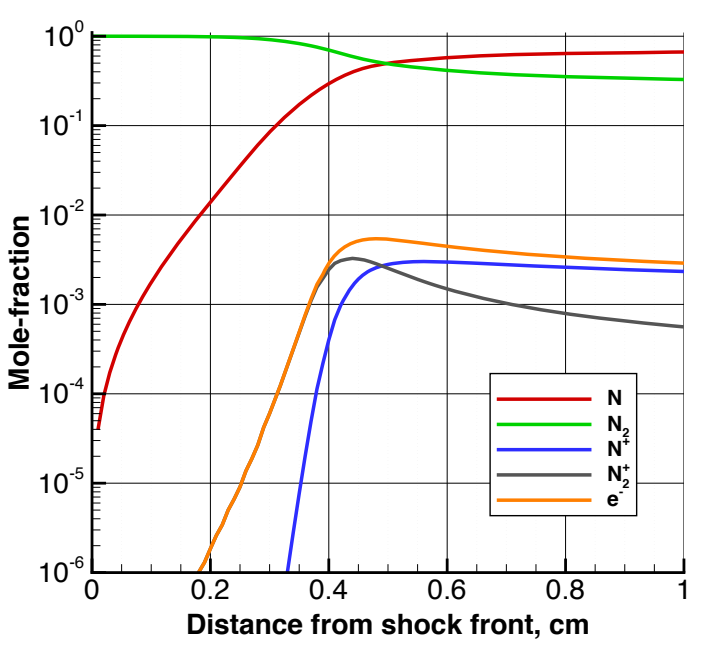

(b) Species mole-fractions obtained with the present model.

Figure 4. Comparisons of the present rotational and electron-electronic-vibrational temperatures and species mole-fractions with the shock-tube experiment by Sakurai et al. ${ }^{9}$

Table 2. Freestream conditions for 1-D post-normal shock calculations.

\begin{tabular}{ccccccc}
\hline \hline Case & $p_{\infty}($ Torr $)$ & $T_{\infty}(K)$ & $\rho_{\infty}\left(\mathrm{g} / \mathrm{cm}^{3}\right)$ & $h_{\infty}(\mathrm{MJ} / \mathrm{kg})$ & $u_{\infty}(\mathrm{km} / \mathrm{sec})$ & $M_{\infty}$ \\
\hline $\mathrm{C} 1$ & 1.30 & 274 & $2.10 \times 10^{-6}$ & 8.79 & 4.0 & 10.86 \\
$\mathrm{C} 2$ & 0.15 & 270 & $2.60 \times 10^{-7}$ & 32.8 & 8.0 & 21.89 \\
$\mathrm{C} 3$ & 0.10 & 300 & $1.497 \times 10^{-7}$ & 50.8 & 10.0 & 25.96 \\
\hline \hline
\end{tabular}

pressure, and these temperatures converge to equilibrium at a distance of about $0.4 \mathrm{~cm}$. In comparing with the measured values, the rotational temperature is almost the same as the measured temperature. However, the electron-electronic-vibrational temperature is larger than the measured value. However, these results show that the present model can still capture the rotational nonequilibrium phenomena behind a strong shock wave. In figure (b), the mole fractions of the various chemical species are presented. The result shows that the relaxations of the rotational and electron-electronic-vibrational modes are mainly generated by $\mathrm{N}_{2}+\mathrm{N}_{2}$ collisions.

In Table 2, initial conditions of several post-normal shock flows are tabulated. The free-stream conditions of cases $\mathrm{C} 1$ to $\mathrm{C} 3$ are constructed to be similar to the high-speed return conditions at altitudes from $45.5 \mathrm{~km}$ to $63.0 \mathrm{~km}$ of the Earth. In the present work, the simplification of nitrogen mixtures without oxygen and radiative heating is adopted to calculate the post-normal shock flows. While this is not an accurate re-entry calculation, it is enough to observe the rotational nonequilibrium of $\mathrm{N}_{2}$ in each re-entry condition.

In Fig. 5, the temperatures and mole-fractions of the post-normal shock calculations are presented. In the $\mathrm{C} 1$ case, the rotational temperature rapidly approaches the translational temperature. Hence, in the $\mathrm{C} 1$ case, the rotational mode can be treated as being in equilibrium with the translational energy. This is the main concept of the two-temperature model. However, for the C2 and C3 cases, rotational and vibrational nonequilibrium are clearly seen. In these cases, the rotational and electron-electronic-vibrational relaxations are almost identical. In figure (b), it is observed that the heavy particle collisions by the dissociated $\mathrm{N}$ significantly affect the rotational and electron-electronic-vibrational relaxations of $\mathrm{N}_{2}$ for the C2 and C3 cases. 


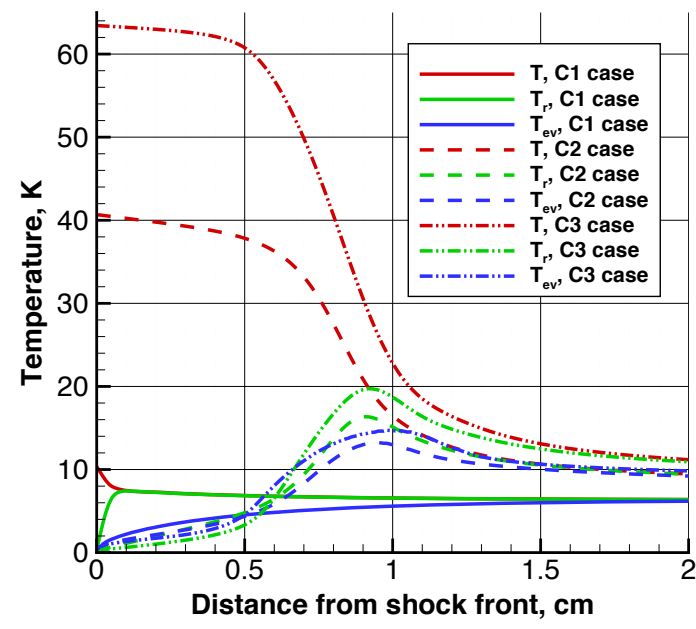

(a) Rotational and electron-electronic-vibrational temperatures.

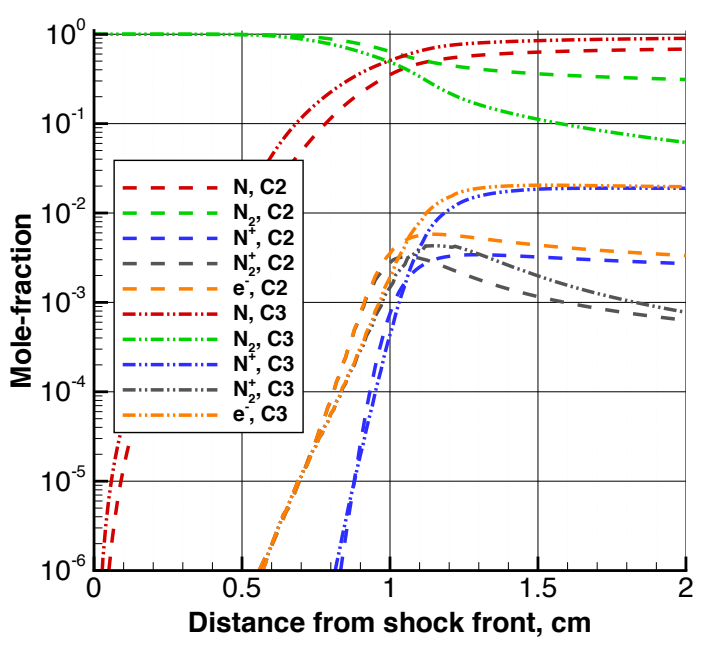

(b) Species mole-fractions.

Figure 5. Post-normal shock flow calculations for various re-entry conditions.

\section{Conclusions}

In order to model rotational nonequilibrium, the rotational mode is separated from the translationalrotational modes of the widely-used two-temperature model. Then, the translational, rotational, and electron-electronic-vibrational modes are considered, and each energy mode is represented by a unique nonequilibrium temperatures. The energy transfers among the energy modes are described by recently evaluated relaxation time parameters including rotation-to-vibration energy transitions. For $\mathrm{N}_{2}+\mathrm{N}$ collisions, the internal energy transfer is treated using state-to-state kinetic methods by coupling the system of master equations. One-dimensional flow equations are constructed with these present thermochemical models, and post-normal shock flow calculations are performed for several existing shock-tube experiments and various re-entry conditions. In comparing with measured rotational and vibrational temperatures, it is shown that the calculated rotational and electron-electronic-vibrational temperatures closely agree with the measured temperatures, and the present thermochemical model is able to capture the rotational nonequilibrium phenomena behind a strong shock wave. In the post-normal shock flow calculation for various re-entry conditions, the present thermochemical model can describe both weak and strong rotational nonequilibrium, and illustrates the possibility of rotational nonequilibrium in high-speed re-entry missions.

\section{Acknowlegement}

The authors gratefully acknowledge funding for this work through Air Force Office of Scientific Research Grants FA9550-11-1-0309 and FA9550-12-1-0483.

\section{References}

${ }^{1}$ Park, C., Nonequilibrium Hypersonic Aerothermodynamics, Wiley, New York, 1990.

${ }^{2}$ Gupta, R. N., Moss, J. N., and Price, J. M., "Assessment of Thermochemical Nonequilibrium and Slip Effects for Orbital Reentry Experiment(OREX)," NASA TM-111600, July 1996.

${ }^{3}$ Furudate, M., Nonaka, S., and Swada, K., Calculation of Shock Shapes over Simple Geometries in Intermediate Hypersonic Air Flow, AIAA 1999-3686, 33rd AIAA Thermophysics Conference and Exhibit, Orlando, Florida, 1999.

${ }^{4}$ Kim, J. G., Kwon, O. J., and Park, C., "Master Equation Study and Nonequilibrium Chemical Reactions for $\mathrm{H}+\mathrm{H}_{2}$ and $\mathrm{He}+\mathrm{H}_{2}$," Journal of Thermophysics and Heat Transfer, Vol. 23, No. 3, 2009, pp. 443-453.

${ }^{5}$ Kim, J. G., Kwon, O. J., and Park, C., "Master Equation Study and Nonequilibrium Chemical Reactions for Hydrogen Molecule," Journal of Thermophysics and Heat Transfer, Vol. 24, No. 2, 2010, pp. 281-290.

${ }^{6}$ Kim, J. G. and Boyd, I. D., "State-resolved thermochemical nonequilibrium analysis of hydrogen mixture flows," Physcis 
of Fluids, Vol. 24, No. 8, pp. 086102, 2012.

${ }^{7}$ Sharma, S. P., and Gillespie, W., Nonequilibrium and Equilibrium Shock Front Radiation Measurements, Journal of Thermophysics and Heat Transfer, Vol. 5, No. 3, 1991, pp. 257-265.

${ }^{8}$ Fujita, K., Sato, S., Abe, T., and Ebinuma, Y., Experimental Investigation of Air Radiation Behind a Strong Shock Wave, Journal of Thermophysics and Heat Transfer, Vol. 16, No. 1, 2002, pp. 77-82.

${ }^{9}$ Sakurai, K., Bindu, V. H., Niinomi, S., Ota, M., and Maeno, K., "CARS Measurement of Vibrational/Rotational Temperatures with Total Radiation Visualization behind Strong Shock waves of $5-7 \mathrm{~km} / \mathrm{s}$," 27th International Symposium on Rarefied Gas Dynamics, AIP Conf. Proc. 1333, 2011, pp. 419-424.

${ }^{10}$ Kim, J. G. and Boyd, I. D., "Master Equation Analysis of Thermochemical Nonequilibrium of Nitrogen," AIAA 2012-3305, 43rd AIAA Thermophysics Conference, New Orleans, Lousiana, 2012.

${ }^{11}$ Jaffe, R., Schwenke, D., Chaban, G., Huo, W., Vibrational and Rotational Excitation and Relaxation of Nitrogen from Accurate Theoretical Calculation, AIAA 2008-1208, 46th AIAA Aerospace Sciences Meeting and Exhibit, Reno, Nevada, 2008.

12 Jaffe, R., Schwenke, D., Chaban, G., Theoretical Analysis of $\mathrm{N}_{2}$ Collisional Dissociation and Rotation-Vibration Energy Transfer, AIAA 2009-1569, 47th AIAA Aerospace Sciences Meeting and Exhibit, Orlando, Florida, 2009.

${ }^{13}$ Park, C., Jaffe, R., and Partridge, H., "Chemical-Kinetic Parameters of Hyperbolic Earth Entry," Journal of Thermophysics and Heat Transfer, Vol. 15, No. 1, 2001, pp. 76-90.

${ }^{14}$ Nishida, M., and Matsumoto, M., "Thermochemical Nonequilibrium in Rapidly Expanding Flows of High-Temperature Air," Zeitschrift fur Naturforschung, Teil A. Physik, Physikalische Chemie, Kosmophysik, Vol. 52, 1997, pp. 358-368.

${ }^{15}$ Gnoffo, P. A., Gupta, R. N., and Shinn, J. L., "Conservation Equations and Physical Models for Hypersonic Air Flows in Thermal and Chemical Nonequilibrium," NASA TP-2867, Feb. 1989.

${ }^{16}$ Park, C. "Rotational Relaxation of $\mathrm{N}_{2}$ Behind a Strong Shock Wave," Journal of Thermophysics and Heat Transfer, Vol. 18, No. 4, 2004, pp. 527-533.

${ }^{17}$ Parker, J. G., "Rotational and Vibrational Relaxation in Diatomic Gases," Physics of Fluids, Vol. 2, No. 4, 1959, pp. 449-462.

${ }^{18}$ Rahn, L. A. and Palmer, R. E., "Studies of Nitrogen Self-Broadening at High Temperature with Inverse Raman Spectroscopy," Journal of Optical Society of America, Vol. 3, No. 9, 1986, pp. 1164-1169.

${ }^{19}$ Millikan, R. C. and White, D. R., "Systematics of Vibrational Relaxation," Journal of Chemical Physics, Vol. 39, No. 12, 1963, pp. 3209-3213.

${ }^{20}$ Appleton, J. P., "Shock-Tube Study of the Vibrational Relaxation of Nitrogen Using Vacuum-Ultraviolet Light Absorption," Journal of Chemical Physics, Vol. 47, No. 9, 1967, pp. 3231-3240.

${ }^{21}$ Allen, R. A., "Nonequilibrium Shock Front Rotational, Vibrational, and Electronic Temperature Measurements," AVCO Everett Research Lab., Everett, MA, Research Rept. 186, Aug. 1964. 